\title{
Fabrication and Fracture Toughness of CNTs/alumina Composites with Fine Microstructures
}

\author{
Naoki Ueda ${ }^{1, a}$, Tomohiko Yamakami ${ }^{1, b}$, Tomohiro Yamaguchi ${ }^{1, c}$, \\ Morinobu Endo ${ }^{1, d}$, Naoto Saito ${ }^{2, e}$ and Seiichi Taruta ${ }^{1, f}$ \\ ${ }^{1}$ Faculty of Engineering, Shinshu University, 4-17-1 Wakasato, Nagano-shi, Nagano, 380-8553 \\ 2Department of Applied Physical Therapy, Shinshu University School of Health Sciences, \\ 3-1-1 Asahi, Matsumoto-shi, Nagano, 390-8621 \\ a 11st307a@shinshu-u.ac.jp, b tyamaka@shinshu-u.ac.jp, c mtmouth@shinshu-u.ac.jp, \\ dendo@endomoribu.shinshu-u.ac.jp, e saitoko@shinshu-u.ac.jp, ${ }^{\dagger}$ staruta@shinshu-u.ac.jp
}

Keywords: Alumina, Carbon nanotubes, Composites, Fracture toughness, Microstructure

\begin{abstract}
Thin carbon nanotubes (CNTs) were heated at $2800^{\circ} \mathrm{C}$ to heighten the crystallinity of the CNTs. The microstructures and fracture toughness of the thin CNTs and the heat-treated thin CNTs/ alumina composites were investigated. Using such thin CNTs was effective for obtaining finer microstructures of the composites but the heat-treatment made the uniform dispersion of the CNTs difficult. The composites containing the heat-treated thin CNTs showed higher fracture toughness than those containing the thin CNTs and the thick CNTs used in previous study when their content was the same.
\end{abstract}

\section{Introduction}

Carbon nanotubes (CNTs) have incredible mechanical properties and high aspect ratio [1-3] so they seem to be superior reinforcement fibers to improve the mechanical properties of ceramic materials. We have combined carbon nanofibers (CNFs) with the average diameter of $104 \mathrm{~nm}$ which were a type of multi-walled CNT with alumina [4,5]. Fracture toughness of the obtained CNFs/alumina composites increased with a decrease in average alumina grain size [5]. This result was explained by bridging and/or pull-out of CNFs bended along alumina grain boundaries. That is, as the average alumina grain size of the composites decreased, the number of bendings per CNF increased, which resulted in an enhancement of the fracture toughness of the composites [5].

On the other hand, if alumina grains in the composites are too fine, CNFs will be only surrounded by many alumina grains and will be regarded as straight or gently curved fibers [5]. In such composites, CNFs will be pulled out more easily and the fracture toughness will not be improved.

Then, we expected that thinner CNTs would bend along
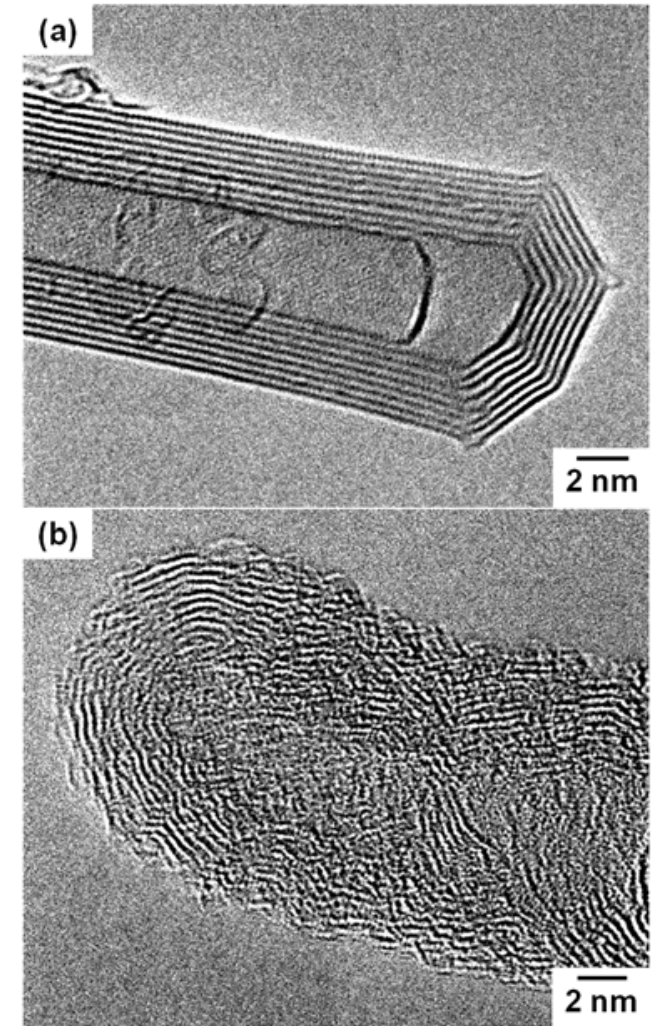

Fig. 1 TEM images of (a) HT-CNTs and (b) pristine CNTs.

alumina grain boundaries even in the composites having much finer microstructures, which would resulted in further improvement in fracture toughness of the composites. In this study, thin multiwalled CNTs were combined with alumina. Because the as-received thin CNTs had a lot of defects and such defects degraded the strength of CNTs [4], the CNTs were heat-treated to heighten the crystallinity. So the microstructure and fracture toughness of the CNTs/alumina composites fabricated using the thin CNTs and the heat-treated thin CNTs were investigated. 


\section{Experimental Procedure}

The average diameter of CNTs (baytubes C150HP, Bayer MaterialScience, Germany) used in this study, which was measured using scanning electron microscope (SEM) photographs, was $18 \mathrm{~nm}$. The pristine CNTs were heat-treated at $2800^{\circ} \mathrm{C}$ for $0.5 \mathrm{~h}$ in $\mathrm{Ar}$ atmosphere to heighten the crystallinity. In addition, the pristine CNTs were acid-treated for $0.5 \mathrm{~h}$ using the acid mixture (conc. $\mathrm{H}_{2} \mathrm{SO}_{4}$ : conc. $\mathrm{HNO}_{3}=3: 1 \mathrm{v} / \mathrm{v}$ ) to disentangle CNT agglomerations and heighten the dispersibility of CNTs. In this paper, the heat-treated CNTs and the acid-treated CNTs are described as HT-CNTs and AT-CNTs, respectively.

HT- and AT-CNTs were dispersed in 2-propanol solution dissolving a small amount of polyvinylbutyral as a dispersant by ultrasonic. Only the 2-propanol solution containing HT-CNTs were jet-milled. After the obtained suspensions were kept quietly for 3 days, the upper layers of them were elutriated to obtain well dispersed CNTs suspensions. The elutriated CNTs suspensions were mixed with high purity alumina powder (TM-DAR, Taimei Chemicals, Japan) by ball-milling for $24 \mathrm{~h}$. The dried powder mixtures containing $1.6 \mathrm{wt} \%$ CNTs were passed through a 100 -mesh sieve and then were consolidated by plasma activated sintering (PAS) at $1350-1400^{\circ} \mathrm{C}$ for $80 \mathrm{~s}$ with uniaxial pressure of $50 \mathrm{MPa}$ in a vacuum. The densification, microstructure and fracture toughness of the obtained composites were estimated by the same method as shown in our previous papers [4,5]. The true density of the CNTs, which was measured by pycnometer method, was $2.0 \mathrm{~g} / \mathrm{cm}^{3}$, which was used for calculation of relative density.

\section{Results and discussions}

Transmission electron microscope (TEM) images of the pristine CNTs and HT-CNTs are shown in Fig. 1. In the photograph, graphene layers of the HT-CNTs showed straight shape and ordered structure, while those of the pristine CNTs showed wave-like shape and disordered structure. The intensity ratios of D-band to G-band in Raman spectra of the pristine CNTs, AT-CNTs and HTCNTs were 1.87, 1.91 and 0.49 , respectively. These results indicate that the amount of defect in the pristine CNTs was decreased by the heat-treatment but was not almost varied by the acid-treatment.

Relative densities of the HT- and AT-CNTs/alumina composites sintered at $1350-1400^{\circ} \mathrm{C}$ were more than $98.6 \%$ and $99.4 \%$, respectively.

Backscattered electron (BSE) images of polished surface of the HT- and AT-CNTs/alumina composites sintered at $1350^{\circ} \mathrm{C}$ are shown in Fig. 2. Each alumina grain was confirmed by the channeling contrast of BSE. The dark areas in the BSE images were either CNTs or pores, but most of them were CNTs because the composites densified to near the full density. Only a few dark areas were observed inside of alumina grains, which indicates that most of the CNTs distributed at alumina 
grain boundaries. The size of almost dark areas in both composites was larger than the diameter of a CNT, and the size of dark areas in the HT-CNTs/alumina composite was larger than that of dark areas in the ATCNTs/alumina composite. These mean that HT- and ATCNTs formed bundles at grain boundaries and HT-CNT bandles were larger than AT-CNT bandles. SEM images of fractured surface of the HT- and AT-CNTs/alumina composites sintered at $1350^{\circ} \mathrm{C}$ are shown in Fig. 3. HTCNTs connected each other and formed network structure around alumina grains as well as AT-CNTs.

Average alumina grain sizes and alumina grain size distributions of the CNTs and CNFs/alumina composites are shown in Fig. 4 and 5, respectively. Average alumina grain sizes of the $1.6 \mathrm{wt} \% \mathrm{CNTs}$ /alumina composites in this study were finer than those of the $1.6 \mathrm{wt} \% \mathrm{CNFs} /$ alumina composite in our previous study [5], which indicates that the CNTs having smaller diameter showed larger retardation effect on the grain growth of alumina.

Though the average alumina grain sizes of the HTand AT-CNTs/alumina composites were almost the same at $1350^{\circ} \mathrm{C}$, the alumina grain size distribution of the HT-CNTs/alumina composite was broader than that of the AT-CNTs/alumina composite. These results and Fig. 2 suggest that the HT-CNTs having a smaller amount of defect were not mixed more uniformly with alumina by convensional ball-milling than AT-CNTs.

At $1400^{\circ} \mathrm{C}$, the average alumina grain size of the HTCNTs/alumina composite was obviously larger than that of the AT-CNTs/alumina composite. The grain size distribution of the HT-CNTs/alumina composite shifted to the larger grain size side, while that of AT-CNTs/ alumina composite did not varied extensively. That is, because the HT-CNTs and their bundles had a smaller amount of defect, they could move with alumina grain boundaries more easily [4]. The other side, the ATCNTs having a larger amount of defect could controll the grain boundary migrations, which resulted in smaller average alumina grain sizes and narrower alumina grain size distributions [4].

Fracture toughness of the HT- and AT-CNTs/alumina composites sintered at $1350^{\circ} \mathrm{C}$ were $5.7 \pm 0.3$ $\mathrm{MPa} \cdot \mathrm{m}^{0.5}$ and $4.5 \pm 0.3 \mathrm{MPa} \cdot \mathrm{m}^{0.5}$, which were higher than that of monolithic alumina $(3.5 \pm 0.1$ $\left.\mathrm{MPa} \cdot \mathrm{m}^{0.5}\right)$. SEM images of Vickers cracks propagated in the HT- and AT-CNTs/alumina composites sintered at $1350^{\circ} \mathrm{C}$ are shown in Fig. 6. In the Vickers cracks of the HT-CNTs/alumina composite, many bridgings and/or pull-outs of CNTs were observed, but in the Vickers cracks of the AT-CNTs/ alumina composite, most CNTs were fractured and bridgings and/or pull-outs of CNTs were rarely observed. Relationship between fracture toughness and average alumina grain size of the $1.6 \mathrm{wt} \%$ HT- and AT-CNTs/alumina composites were ploted in the figure of the relationship obtained for the 0.4-2.5 wt\% CNFs/alumina composites in previous study [5], which are shown in Fig. 7. The relationship of the HT-CNTs/alumina composite was almost on the curve drawn for that of the CNFs/ alumina composites. And the fracture toughness of the HT-CNTs/alumina composite were higher than that of the $1.6 \mathrm{wt} \% \mathrm{CNFs} /$ alumina composite because the average alumina grain sizes in the HTCNTs/alumina composite were finer than those in the CNFs/alumina composite. However, drastic 
improvement in fracture toughness was not achieved even using thinner CNTs due to the formation of many larger HT-CNT bundles. So if individual HT-CNTs distribute much more uniformly at alumina grain boundaries of the composites, the composites having much finer microstructure will be obtained and the further improvement in fracture toughness will be achieved. On the other hand, though the AT-CNTs distributed more uniformly than the HT-CNTs, fracture toughness of the AT-CNTs/alumina composites were lower and deviated from the curve in Fig. 7 because AT-CNTs having the large amount of defects were fractured easily during the crack propagations.

\section{Summary}

Thin CNTs with average diameter of $18 \mathrm{~nm}$, which had a large amount of defect, were heated at $2800^{\circ} \mathrm{C}$, which caused the decrease in defect content. Consequently, the heat-treated CNTs (HT-CNTs) could not be mixed more uniformly with alumina powder, formed larger bundles in the obtained composites and showed smaller retardation effect on the grain growth of alumina, particularly at $1400^{\circ} \mathrm{C}$, than AT-CNTs which were obtained by acidtreating the thin CNTs and had a large amount of defect. The microstructures and fracture toughnesses of the HTCNTs/alumina composite were finer and higher than those of the CNFs/alumina composite in previous study. However, drastic improvement in fracture toughness was not achieved even using such HT-CNTs due to the formation of many larger bundles. The other side, while the microstructres of the AT-CNTs/alumina composite were finer and more uniform, particularly at $1400^{\circ} \mathrm{C}$, the fracture toughnesses were much lower because AT-CNTs were fractured easily during the crack propagations.

Acknowledgement The authors thank Dr. K. Fujisawa of Endo Lab. in Shinshu Univ. for providing TEM images.

\section{References}

[1] T. Hayashi, Y. A. Kim, T. Natsuki and M. Endo, Mechanical properties of carbon nanomaterials. Chem. Phys. Chem. 8 (2007) 999-1004.

[2] M. F. Yu, O. Lourie, M. J. Dyer, K. Moloni, T. F. Kelly and R. S. Ruoff, Strength and Breaking Mechanism of Multiwalled Carbon Nanotubes Under Tensile Load. Science, 287 (2000) 637-640.

[3] A. H. Barber, I. K. Ashiri, S. R. Cohen, R. Tenne and H. D. Wagner, Stochastic strength of nanotubes: An appraisal of available data. Compos. Sci. Technol., 65 (2005) 2380-2384.

[4] N. Ueda, T. Yamakami, T. Yamaguchi, K. Kitajima, Y. Usui, K. Aoki, M. Endo, N. Saito and S. Taruta, Microstructure development and fracture toughness of acid-treated carbon nanofibers/ alumina composites. J. Ceram. Soc. Japan. 120 (2012) 560-568.

[5] N. Ueda, T. Yamakami, T. Yamaguchi, K. Kitajima, Y. Usui, K. Aoki, T. Nakanishi, F. Miyaji, M. Endo, N. Saito and S. Taruta, Fabrication and mechanical properties of high-dispersiontreated carbon nanofiber/alumina composites. J. Ceram. Soc. Japan. 118 (2010) 847-854. 